\title{
Analyzing Gas Diffusion in LXe-TPCs for Upcoming Hydrogen Doping Studies
}

\author{
Malena Fassnacht, University of Houston - SULI Program
}

Hugh Lippincott, Fermilab

\section{Introduction}

Liquid xenon time projection chambers (LXe-TPCs) currently lead the direct-detection search for dark matter (DM) in the form of weakly interacting massive particles (WIMPs). However, sensitivity is currently limited to DM with masses above 5 $\mathrm{GeV} / \mathrm{c}^{2}$. Hydrogen doping in LXe-TPCs would allow for better kinematic matching between light DM particles and target nuclei, expanding sensitivity to DM masses down to $200 \mathrm{MeV} / \mathrm{c}^{2}$. Additionally, this method would introduce sensitivity to spindependent DM. So far, no major experiments have explored this parameter space [1].

Plans are underway to dope an existing small-scale LXe-TPC (XELDA) with $\mathrm{H}_{2}$ to study behavior and mixing properties in LXe. This will include measuring the Henry coefficient and characterizing the charge-to-light spectrum of $\mathrm{H}_{2}$ recoils in LXe. Prior to this, it is important to determine an expected time scale of $\mathrm{H}_{2}$ diffusion and saturation in our TPC.

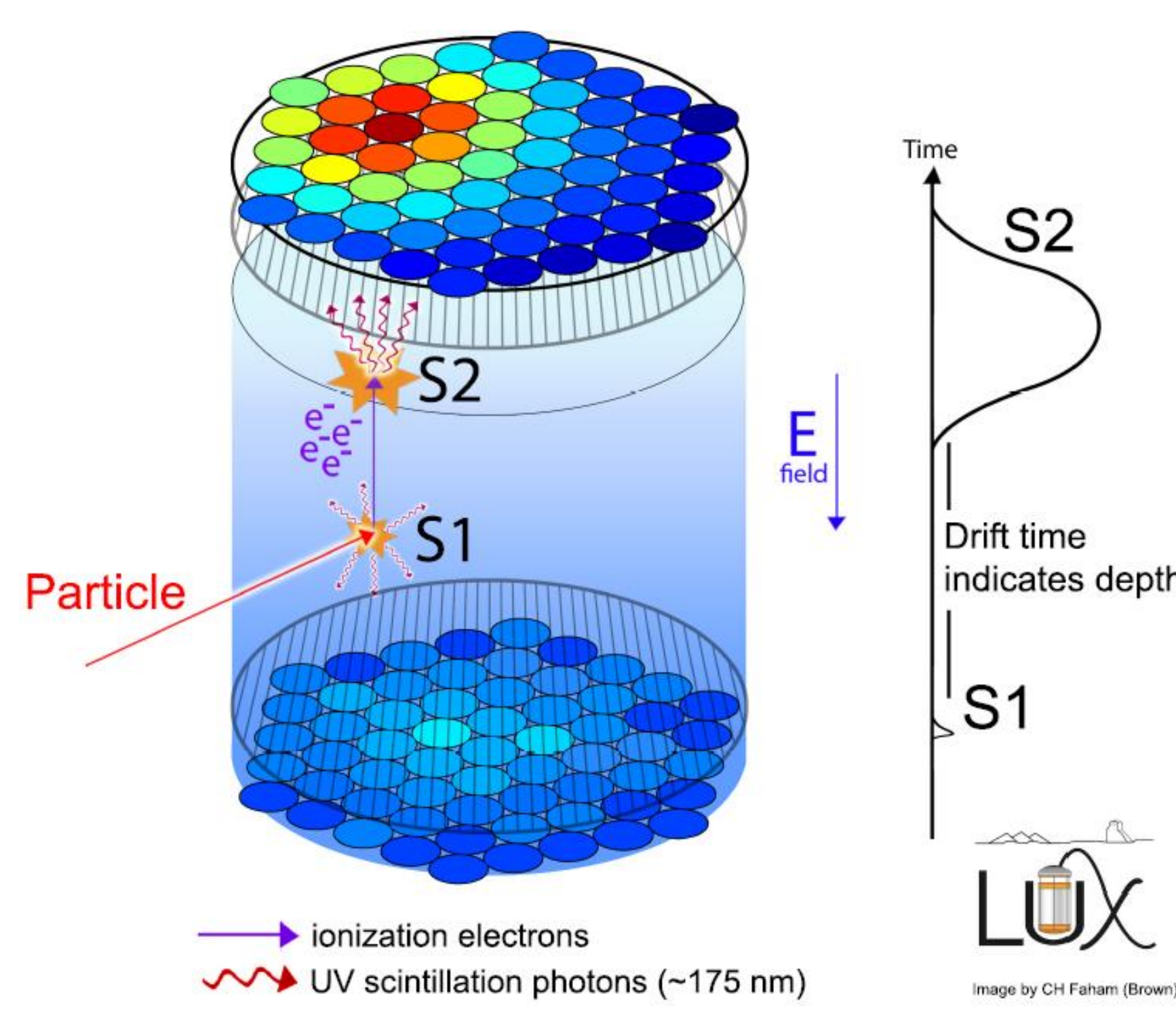

Fig 1. Diagram of an LXeTPC. A WIMP interacts with a $\mathrm{Xe}$ nucleus, producing scintillation light (S1) and ionized electrons. An electric field drifts the charges to a liquid-gas interface, where they are extracted and produce a secondary sillation signal (S2)

\section{Methodology}

Tritiated methane $(\mathrm{CH} 3 \mathrm{~T})$ is a radioactive gas with mixing properties similar to $\mathrm{H}_{2}$. In this project, $\mathrm{CH} 3 \mathrm{~T}$ is used to understand diffusion and equilibration of non-condensable gases in the XELDA detector.

\section{Detector Operations}

- Injected CH3T into source panel, then switched detector into forced circulation mode to encourage Xe-CH3T mixing

- Returned to data acquisition after four hours of circulation and collected 12 ten-minute data sets.

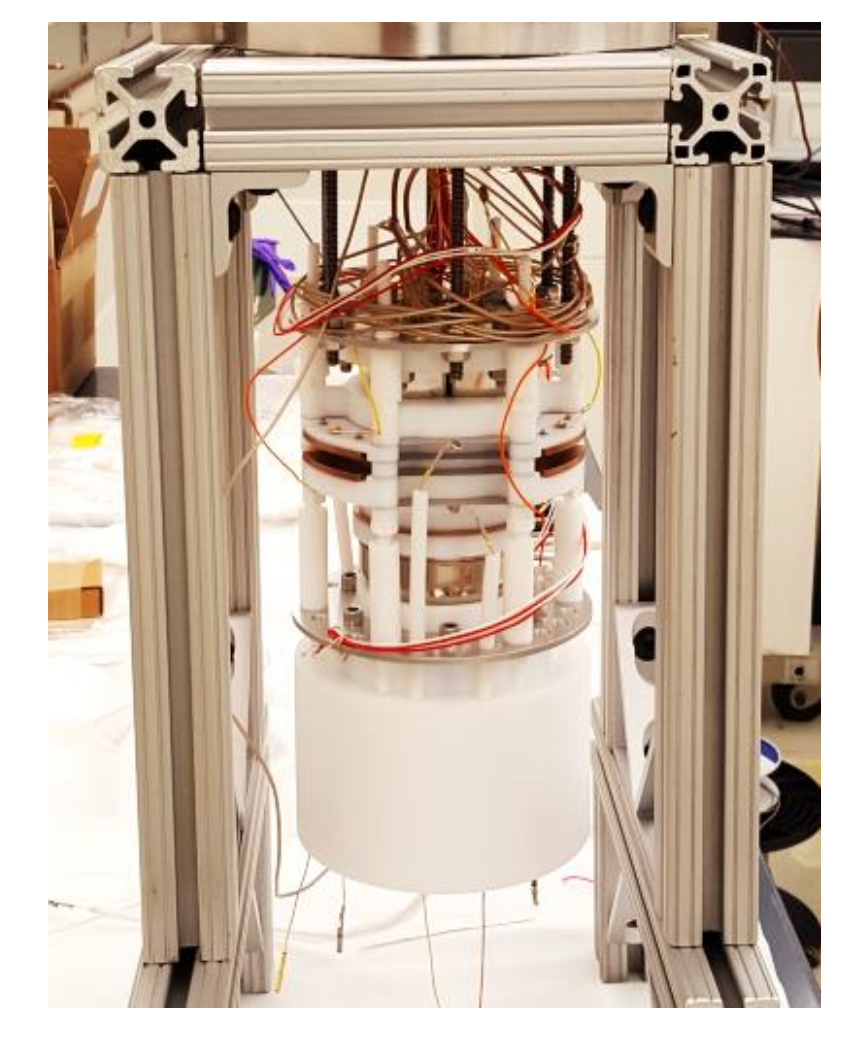

Fig 2. The XELDA LXe-TPC.

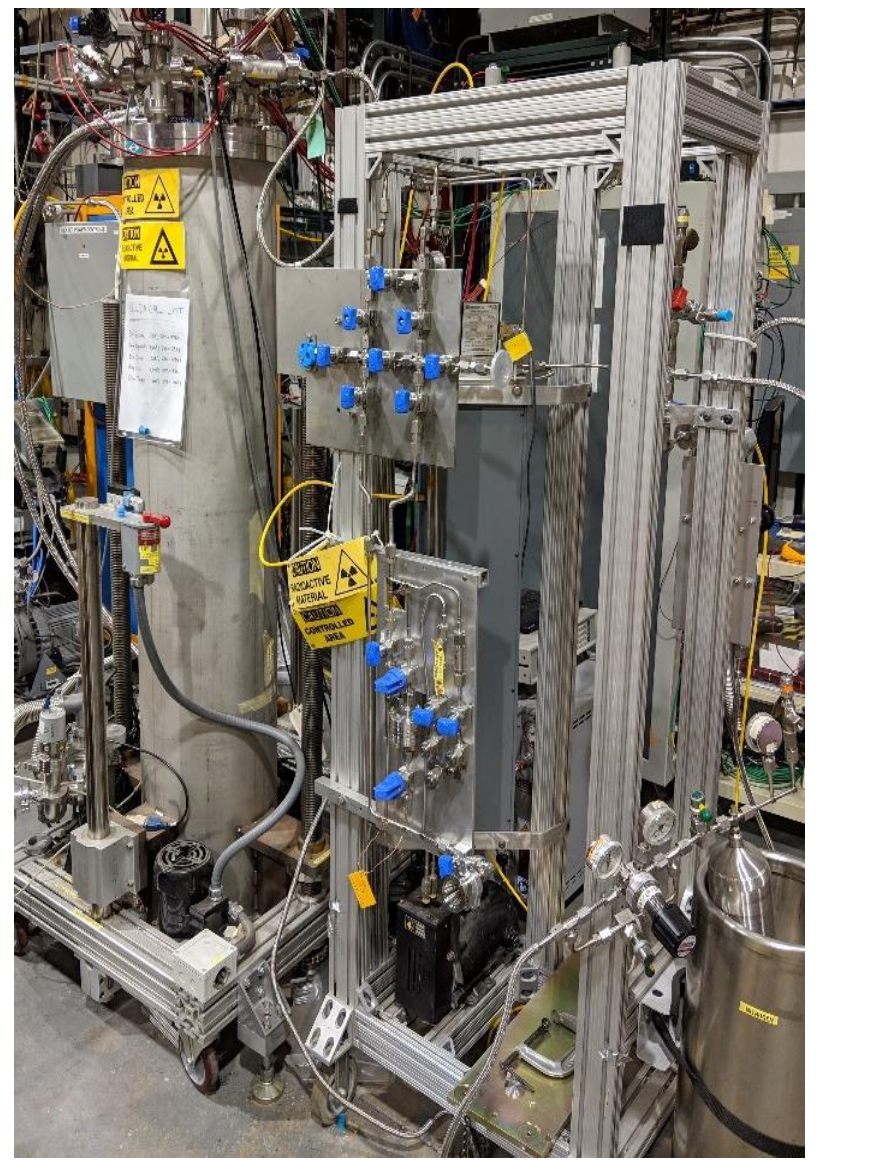

Fig 3. The full XELDA detector setup.

\section{Data Analysis}

- Processed and analyzed raw data using ROOT.

- Calculated overall trigger rate for each ten-minute data set to track CH3T activity in the LXe.

Used S1 pulse area to discriminate between tritium beta decay and 'other' events, then analyzed rate trends separately.

\section{Rate Analysis Results}

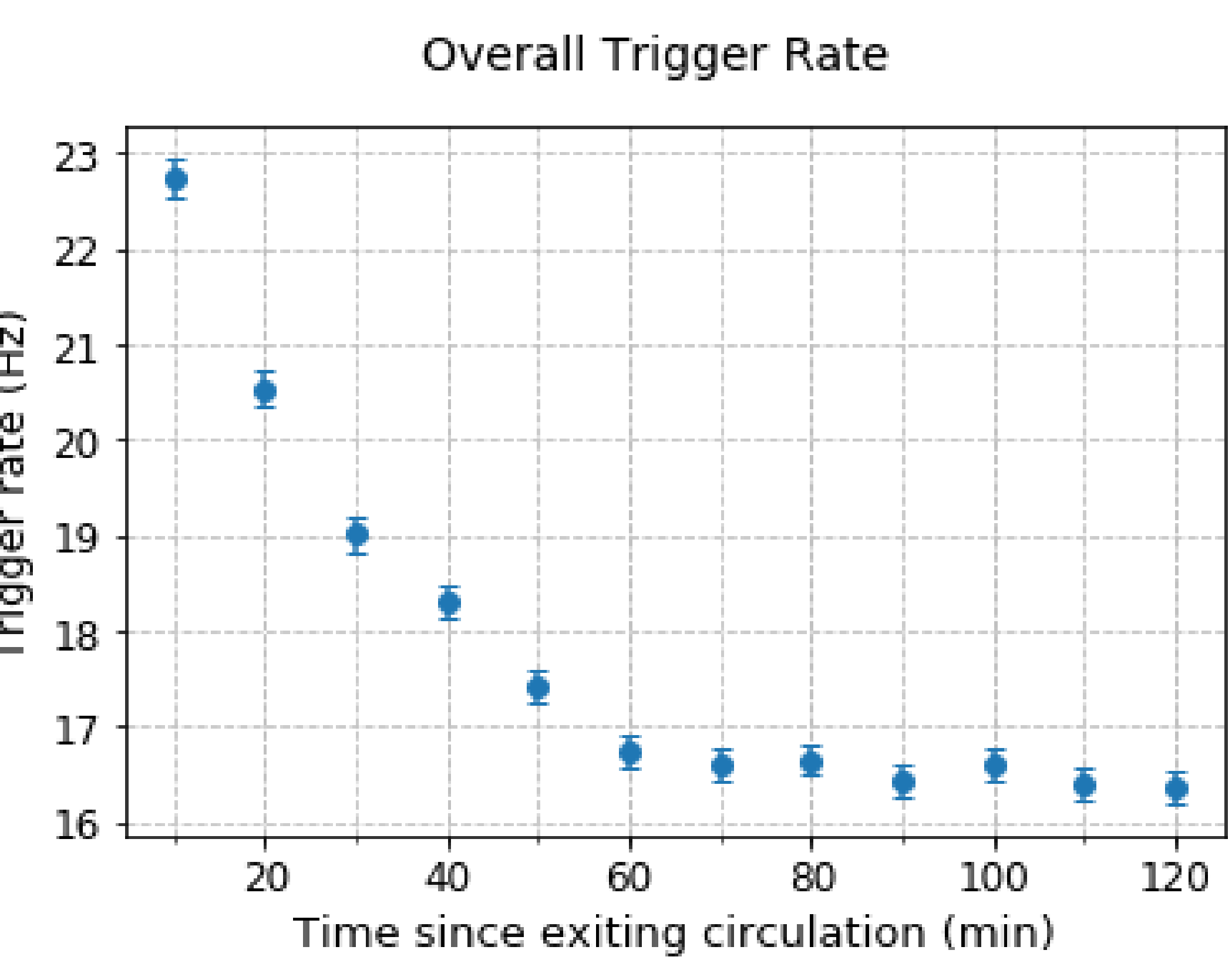

Fig 4. Overall trigger rate following four hours of circulation. The rate falls from $22.7 \mathrm{~Hz}$ to $16.4 \mathrm{~Hz}$ over a two-hour period, with stabilization beginning appx. one hour after exiting circulation.

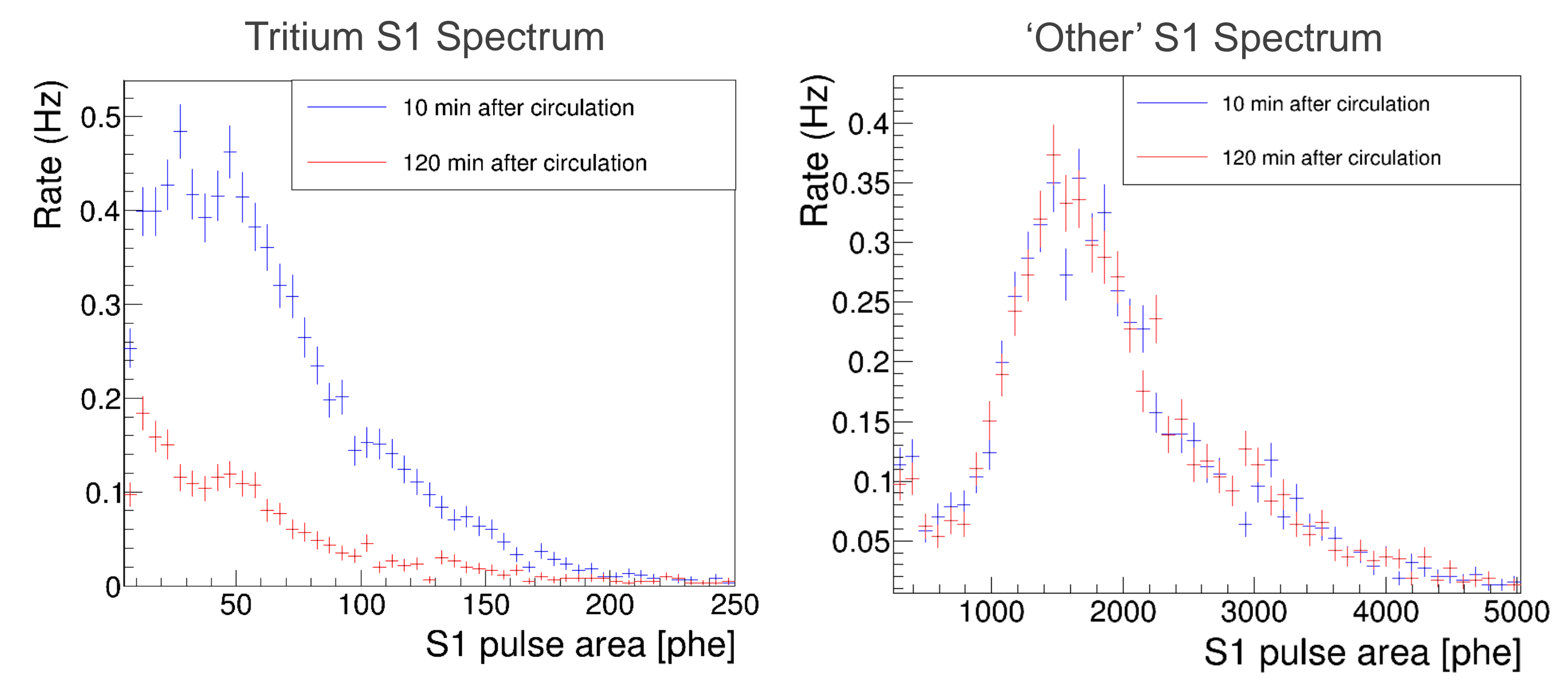

Fig 5. Comparison of rate-normalized S1 spectrums for tritium beta decay events (left) and all other events (right) before and after trigger rate stabilization. Two hours after circulation, the tritium rate fell significantly, whereas the rate of all other events remained constant. This confirms the trend in overall trigger rate was due to a decrease in $\mathrm{CH} 3 \mathrm{~T}$ concentration in the LXe.
The CH3T over-saturated the LXe during the four-hour circulation period and came into equilibrium approximately one hour after exiting circulation mode. Tritium decay events are still observed after rate stabilization, indicating that a measurable amount of $\mathrm{CH} 3 \mathrm{~T}$ remained in solution after equilibration.

\section{$\mathrm{H}_{2}$ Afterpulsing Calculation}

Light gases can permeate PMT window materials over time, causing detrimental afterpulsing effects. This is illustrated by the results of an ET Enterprises study, which found that exposure to $\mathrm{He}$ at $1 \mathrm{~atm}$ causes significant afterpulsing after $17 \mathrm{~min}$ and PMT failure after 3 hours in a model 9266B PMT [2].

The pressure inside a PMT at time $t$ is given by Eqs. (1) and (2). To find the permeability $K$, the data sets in Fig. 6 were extrapolated to $T=194 \mathrm{~K}$. $P_{\text {int }}$ was then calculated for XELDA's top 1" PMTs (HM R850) at $T=194 \mathrm{~K}$ and $P_{\text {ext }}=1 \mathrm{~atm}$.

Fig 6. Fits to experimental data from Lee et al. [3], Kurita et al. [4], Altemose, and Norton [5], for $K_{\mathrm{H} 2}$ in fused silica glass $(1000 \mathrm{~K}<\mathrm{T}<455 \mathrm{~K})$.

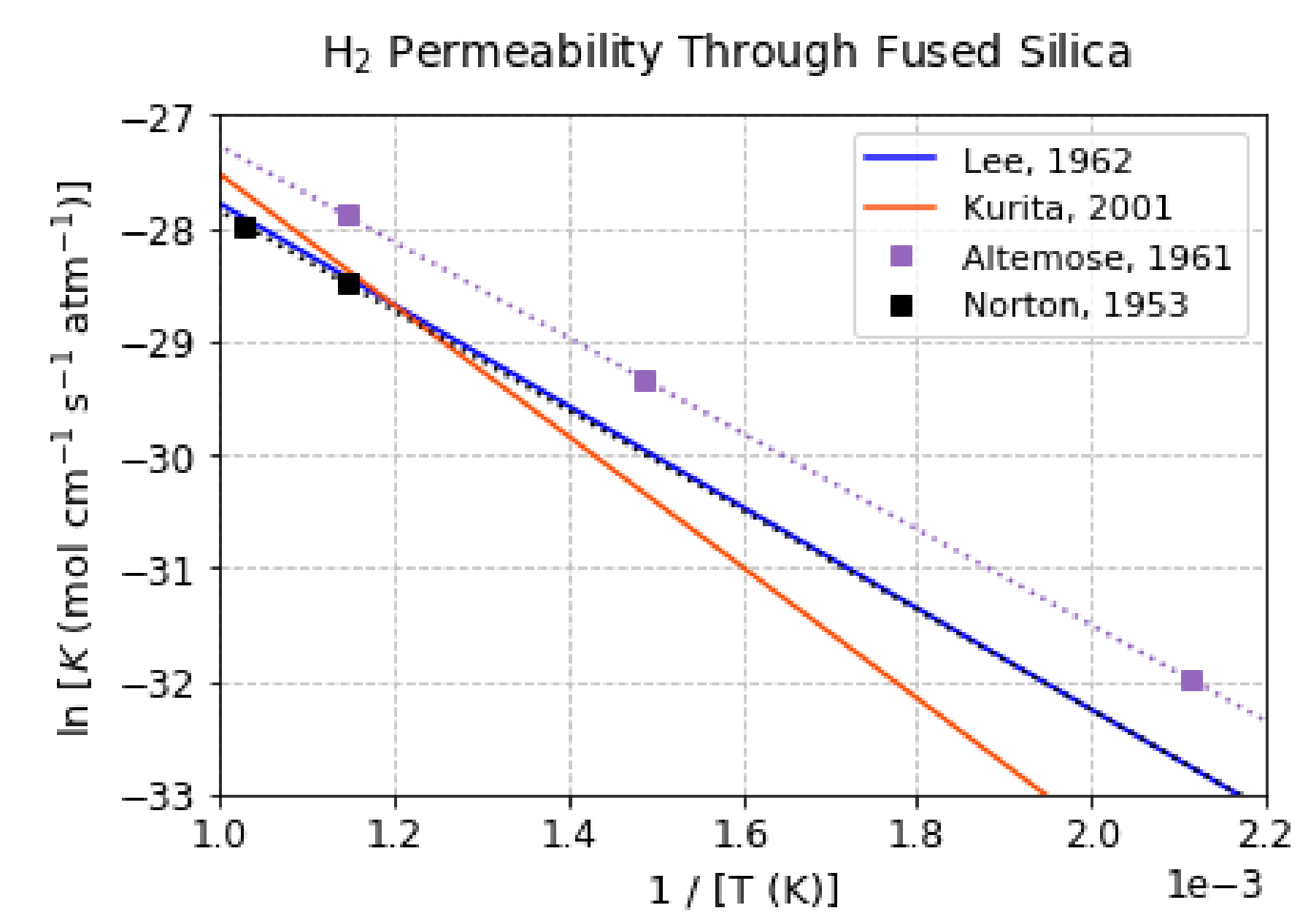

$$
\begin{aligned}
& P_{\text {int }}=C t P_{\text {ext }} /(1+C t) \\
& C=R T K A /(V d)
\end{aligned}
$$

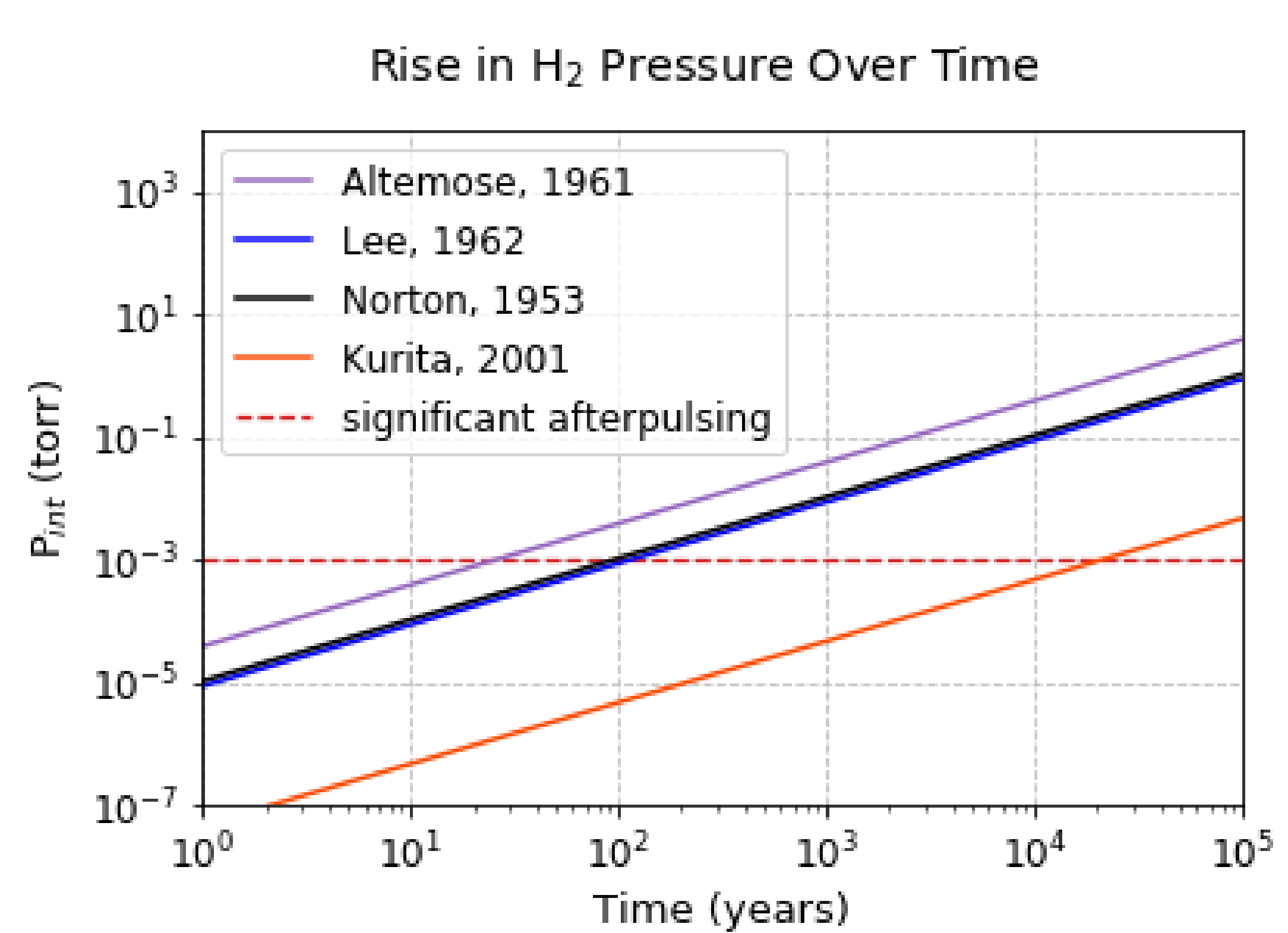

Fig 7. $\mathrm{H}_{2} P_{\text {int }}$ over time at $T=194 \mathrm{~K}$ and $P_{\text {ext }}=1$ atm. Our PMTs are projected to reach the significant afterpulsing threshold (10-3 torr) after appx 26 yrs, 94 yrs, 115 yrs, and 19,000 yrs using permeability data from Altemose, Noton,

\section{Conclusions}

- Using our circulation methods, the CH3T saturated the LXe on a reasonable experimental time scale and remained in solution after circulation. We expect $\mathrm{H}_{2}$ to display similar mixing behavior, presenting encouraging results for future operations.

- It is projected that $\mathrm{H}_{2}$ will not cause significant afterpulsing in our PMTs until at least 26 years of exposure. Based on this analysis, it is unlikely that detrimental effects will occur within the time frame of our hydrogen operations.

\section{References}

[1] D. Temples, Low-mass dark matter reach in LXe-TPCs using hydrogen," (2018).

[2] ET Enterprises Limited, "The effect of exposure to helium on photomultiplier performance and lifetime," R/P101 Issue 1 (2012).

[3] R.W. Lee et al. "Diffusion of Hydrogen and Deuterium in Fused Quartz," J. Chem. Phys. 36, 1062 (1962).

N. Kurita et al. "Measurements of hydrogen permeation through fused silica and borosilicate glass by electrochemical pumping using oxide protonic conductors, Solid State lonics 146, no. 1-2, (2002): 101-111.

[5] V. O. Altemose, "Helium Diffusion through Glass," Journal of Applied Physics 32, 1309 (1961). 